\title{
The effect of perceived distance on perceived movement*
}

\author{
WALTER C. GOGEL and JEROME TIETZ \\ University of California, Santa Barbara, California 93106
}

\begin{abstract}
Equations were developed to predict the apparent motion of a physically stationary object resulting from head movement as a function of errors in the perceived distances of the object or of its parts. These equations, which specify the apparent motion in terms of relative and common components, were applied to the results of two experiments. In the experiments, the perceived slant of an object was varied with respect to its physical slant by means of perspective cues. In Experiment I, O reported the apparent motion and apparent distance of each end of the object independently. The results are consistent with the equations in terms of apparent relative motion, but not in terms of apparent common motion. The latter results are attributed to the tendency for apparent relative motion to dominate apparent common motion when both are present simultaneously. In Experiment II, a direct report of apparent relative motion (in this case, apparent rotation) was obtained for illusory slants of a physically frontoparallel object. It was found that apparent rotations in the predicted direction occurred as a result of head motion, even though under these conditions no rotary motion was present on the retina.
\end{abstract}

A physically stationary object viewed while moving the head can appear stationary despite displacement of its image on the retina or despite changes in eye position required to maintain fixation on the object (Wallach \& Kravitz, 1965). This has been attributed to a compensating process in which the $O$ takes his head movement into account in evaluating the changes in the retinal stimulus or in eye rotations (von Holst, 1954; Rock, 1966). Such compensation theories postulate a central mechanism in which retinal image displacement or the eye movements necessary to maintain fixation are compared with information regarding $O$ 's head movement. However, information about head movement and retinal displacement or eye rotation is not sufficient to determine the perception of the object as stationary. This is illustrated in Fig. 1, in which the upper drawing shows $\mathrm{O}$ moving his head from right to left through a distance, $\mathrm{A}$, with the physical change in the direction from $\mathrm{O}$ of a stationary object, $\mathrm{f}$, located at a physical distance, $D_{\mathrm{f}}$, given by $\phi_{1}+\phi_{2}=\phi_{\mathrm{T}}$. Perceived characteristics are indicated by the prime notation in the lower drawing, with $\mathrm{m}^{\prime}$ the magnitude of perceived movement. The given combination of $\mathrm{A}^{\prime}$ and $\phi_{\mathrm{T}}$ can be produced by a perceptually stationary object perceptually at $D_{\mathbf{f}}^{\prime}$ or by an object perceptually at $D_{e}^{\prime}$, with an apparent motion, $m_{e}^{\prime}$, in the same direction as $O$ 's head or by an object perceptually at $\mathrm{D}_{\mathrm{g}}^{\prime}$, with an apparent motion, $\mathrm{m}_{\mathrm{g}}^{\prime}$, in the direction opposite to $\mathrm{O}^{\prime} \mathrm{s}$ head movement. It follows that for a given value of $A^{\prime}$ and $\phi_{\mathrm{T}}^{\prime}, \mathrm{O}$ 's perception of whether the object is moving in a direction with or against the movement of his head or is stationary is related to the perceived distance of the object.

Evidence that the perceived distance of the object is a factor in determining the apparent movement of objects

\footnotetext{
*This investigation was supported by U.S. Public Health Service Research Grants NS 08883, from the National Institute of Neurological Diseases and Stroke, and MH 15651, from the National Institute of Mental Health.
}

associated with head movement is discussed by Ittelson (1960). Further evidence is found in a study by Hay and Sawyer (1969), in which the introduction of a difference between the convergence and physical distance of a spot of light viewed with a moving head required a physical movement of the light in a direction consistent with Fig. 1 in order for the object to appear stationary. Wallach, Yablick, and Smith (1972) varied both the accommodation and convergence to the spot independently of its physical distance and found still larger effects. In agreement with these results, Gogel and Tietz (1972) found that the perception of movement of
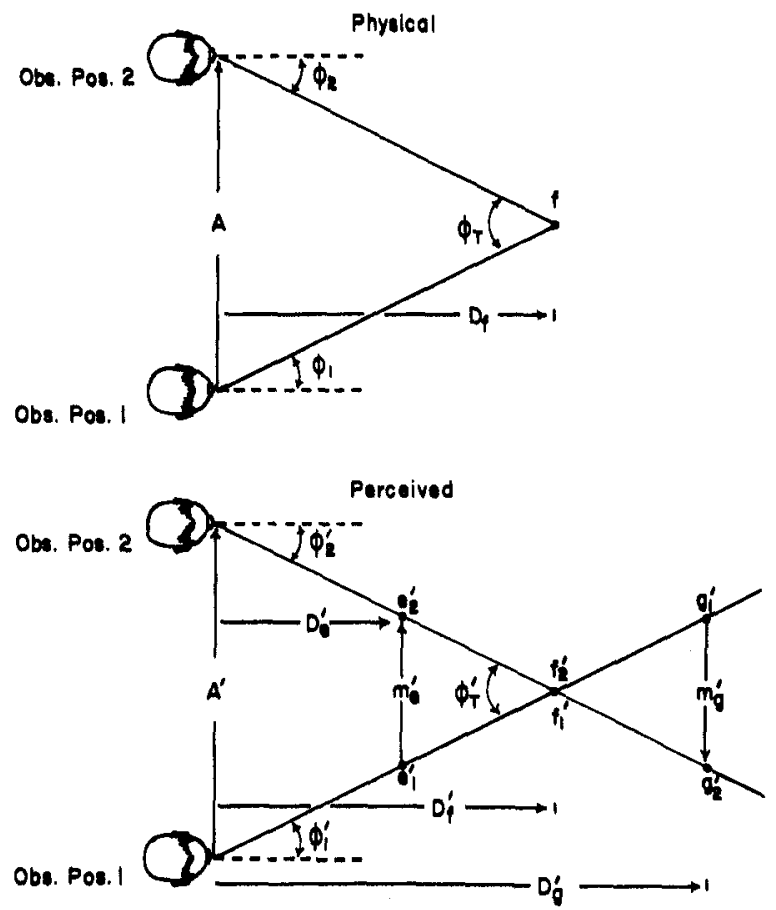

Fig. 1. Factors involved in the perception of motion of a physically stationary point (f) as a result of laterally moving the head from Position 1 to Position 2. 


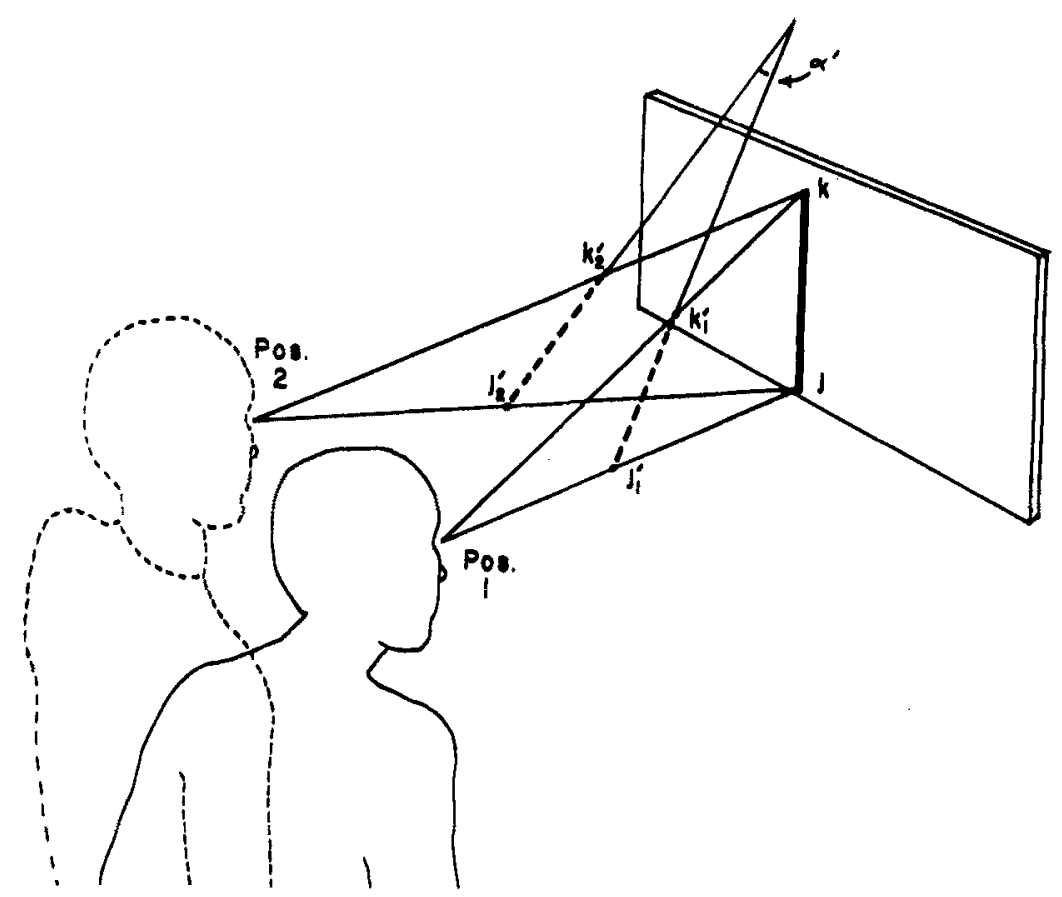

Fig. 2. A schematic diagram illustrating a difference between a physical (jk) and an apparent $\left(j^{\prime} k^{\prime}\right)$ orientation of an object as viewed from two positions (1 and 2 ).

a physically stationary point of light viewed with lateral head movement varied predictably as a function of the difference between the physical distance of the point and its perceived distance.

The expected effect of a difference between physical distance, $D$, and perceived distance, $D^{\prime}$ upon the perception of motion, $m^{\prime}$ associated with lateral head movement can be applied to the case in which more than one physically stationary point are viewed simultaneously. This is illustrated in the perspective drawing of Fig. 2 for the situation in which the head is moved laterally between two positions (Position 1 and Position 2) while viewing the two points, " $j$ " and " $k$ " (extended Object $\mathrm{jk}$ ), with Point $\mathrm{k}$ vertically displaced above Point j. The portion of the drawing in Fig. 2 showing the physical plane of the stimulus, $\mathrm{jk}$, is for illustrative purposes only, with Object jk the only stimulus present in an otherwise dark visual field.

In the situation illustrated in Fig. 2, Points $\mathrm{j}$ and $\mathrm{k}$ are physically stationary and at the same physical distance from $O$. Point $j$ is at the same perceived distance $\left(D_{j}^{\prime}\right)$ from $O$ whether viewed from Position 1 or Position 2 $\left(D_{j_{1}}^{\prime}=D_{j_{2}}^{\prime}\right)$. Also, Point $k$ is at the same perceived distance $\left(D_{k}\right)$ from $O$ whether viewed from Position 1 or Position $2\left(D_{k_{1}}^{\prime}=D_{k_{2}}^{\prime}\right)$, but $D_{j}^{\prime}$ is less than $D_{k}^{\prime}$.

As $O$ moves his head from Position 1 to Position 2, Point $\mathrm{j}$ has an apparent motion $\left(\mathrm{m}_{\mathrm{j}}^{\prime}\right)$ from $\mathrm{j}_{1}^{\prime}$ to $\mathrm{j}_{2}^{\prime}$ and Point $\mathrm{k}$ has an apparent motion $\left(\mathrm{m}_{\mathrm{k}}^{\prime}\right)$ from $\mathrm{k}_{1}^{\prime}$ to $\mathrm{k}_{2}^{\prime}$. In this study, the apparent motion of a point is designated as positive if it is in the same direction as the head motion and as negative if it is in a direction opposite to the head motion. Figure 2 illustrates a situation in which $\mathrm{D}_{\mathrm{j}}>\mathrm{D}_{\mathrm{j}}^{\prime}$ and $\mathrm{D}_{\mathrm{k}}>\mathrm{D}_{\mathrm{k}}^{\prime}$ and the apparent motion of both points is in the direction of the head motion $\left(\mathrm{m}_{\mathbf{j}}^{\prime}\right.$ and $\mathrm{m}_{\mathbf{k}}^{\prime}$ are both positive). This apparent motion of Points $j$ and $k$ occurs in the apparent plane defined by $j_{1}^{\prime} j_{2}^{\prime} k_{2}^{\prime} k_{1}^{\prime}$, with this apparent plane differing in orientation from the plane of orientation of the physical stimulus, $\mathrm{jk}$.

The total apparent motions, $m_{j}^{\prime}$ and $m_{k}^{\prime}$, resulting from head motion can be analyzed into two components. One component (called apparent relative motion) is defined as the difference in apparent motion $\left(m_{j}^{\prime}-m_{k}^{\prime}\right)$ between Points $j$ and $k$. This component of the total apparent motion produces an apparent rotation of Object jk, indicated by $\alpha^{\prime}$ in Fig. 2, as the head is moved between Positions 1 and 2. The other component of the perceived motion (called apparent common motion) is the apparent motion that $j$ and $k$ have in common. In Fig. 2, this component is the common lateral displacement of $\mathrm{jk}$ in the apparent plane, defined by $j_{1}^{\prime} j_{2}^{\prime} k_{2}^{\prime} k_{1}^{\prime}$, as the head is moved between Positions 1 and 2. A lateral component of apparent motion common to both points will oecur if and only if $m_{j}^{\prime}$ and $m_{k}^{\prime}$ are both different from zero and have the same sign. The magnitude of the apparent common motion is specified by the apparent motion of the point having the smaller absolute value of apparent motion (in this case, the apparent motion, $\mathrm{m}_{\mathrm{k}}^{\prime}$, between $\mathrm{k}_{1}^{\prime}$ and $\mathrm{k}_{2}^{\prime}$ ). Together, the perceived relative motion and the perceived common motion specify the entire perceived motion of Object $\mathrm{jk}$ resulting from head motion.

Consistent with Fig. 1, the relation between the perceived movement and the perceived distance of any point is expressed by

$$
\mathrm{m}^{\prime}=\mathbf{A}^{\prime}-\phi_{\mathrm{T}}^{\prime} \mathrm{D}^{\prime}
$$

where $\mathrm{m}^{\prime}$ is the perceived motion of the point associated 
with the sensed head motion, $A^{\prime}, D^{\prime}$ is the perceived distance of the point from $O$, and $\phi_{T}^{\prime}$ is the sensed change in the direction of the point from $\mathrm{O}$ expressed in radians (Gogel \& Tietz, 1972). It was found in the study by Gogel and Tietz that the direction of the motion of a single point of light could be predicted by assuming that $\mathrm{A}^{\prime}=\mathrm{A}$ and $\phi_{\mathrm{T}}^{\prime}=\phi_{\mathrm{T}}$. In this case, Eq. 1 becomes

$$
m^{\prime}=A\left(D-D^{\prime}\right) / D \text {. }
$$

Equation 2 can be applied to the situation in which the two points, $\mathbf{j}$ and $k$, are presented simultaneously. It follows from Eq. 2 that

$$
m_{j}^{\prime}-m_{k}^{\prime}=A\left(\frac{D_{k}^{\prime}}{D_{k}}-\frac{D_{j}^{\prime}}{D_{j}}\right),
$$

with $m_{j}^{\prime}-m_{k}^{\prime}$ the apparent relative motion of $j$ and $k$. According to Eq. 3, apparent relative motion will occur whenever $D_{k}^{\prime} / D_{k} \neq D_{j}^{\prime} / D_{j}$, or $m_{j}^{\prime} \gtrless m_{k}^{\prime}$ whenever $\mathrm{D}_{\mathrm{k}}^{\prime} / \mathrm{D}_{\mathrm{k}} \gtrless \mathrm{D}_{\mathrm{j}}^{\prime} / \mathrm{D}_{\mathrm{j}}$. On the other hand, apparent common motion will occur whenever $m_{\mathbf{j}}^{\prime}$ and $m_{\mathbf{k}}^{\prime}$ are both different from zero and have the same sign. According to Eq. 2, this will occur only if both $D_{j}-D_{j}^{\prime}$ and $D_{k}-D_{k}^{\prime}$ both differ from zero and have the same sign. These relations between apparent relative and common motion and perceived distance apply to all orientations of the stimulus object, $\mathrm{jk}$, and not only to the case illustrated in Fig. 2, in which $\mathrm{j}$ and $\mathrm{k}$ are physically at the same distance from $O$. The purpose of the present study is to determine whether the verbal report of the perceived motion of Parts $\mathrm{j}$ and $\mathrm{k}$ of the stimulus resulting from head motion can be predicted from the perceived (and physical) distances of these parts using Eqs. 2 and 3. In Experiment $\mathrm{I}$, the apparent motion of $\mathrm{j}$ and $\mathrm{k}$ were reported separately. It is reasonable to assume that if the separate reports of the magnitude of the motion of $j$ and $\mathrm{k}$ are different, $\mathrm{j}$ and $\mathrm{k}$ would have been reported as having a relative motion if a direct report of relative motion had been obtained. Also, in Experiment $\mathrm{I}$, if each of the separate reports of motion of $j$ and $k$ have the same sign and are different from zero, it will be assumed that common motion would have been reported if a direct report of the common motion had been obtained. In Experiment II, unlike Experiment I, direct judgments of apparent relative motion were obtained by asking for reports of the apparent rotation of the object ( $\alpha^{\prime}$ in Fig. 2).

Perceived distance in the present study was measured by verbal reports. It has been found in earlier studies (Gogel, 1969; Gogel \& Tietz, 1972) that in situations in which there are many cues to distance, Os give verbal reports which are approximately .6 of the physical distance. This tendency to underestimate verbally the distance of objects in stimulus-rich environments is assumed to indicate that in reporting apparent distance $O$ overestimates in memory the magnitude of a foot ruler, with this overestimation applying to visually reduced as well as to visually rich conditions of observation. If it is assumed that perceived distance is equal to physical distance in environments with many cues to distance, the relation between reported and physical distance in such environments can be used to transform the reports of distance $\left(D_{v}\right)$ obtained under reduced conditions to perceived distance $\left(\mathrm{D}^{\prime}\right)$. For this purpose, verbal reports of the apparent distances of objects located in a calibration field containing many visual cues of distance were obtained. From the assumption that distances in this calibration field were correctly perceived, a relation between verbal reports and perceived distances (a calibration equation) was determined for each $\mathrm{O}$ and applied to the verbal reports of the distances of Points $\mathrm{j}$ and $\mathrm{k}$ presented under reduced conditions, so as to transform these verbal reports to perceived distances. The need to calibrate verbal reports of distance is clear in attempting to evaluate common motion, since the sign as well as the magnitude of $D-D^{\prime}$ sometimes can differ if $D_{v}$ is mistakenly used in place of $\mathrm{D}^{\prime}$. It is of less importance to calibrate reports of the extent of motion, $\mathrm{m}^{\prime}$, since the sign of $m^{\prime}$ in Eq. 2 and the sign of $m_{j}^{\prime}-m_{k}^{\prime}$ in Eq. 3 is unaffected by an $O$ constant of calibration in measuring $\mathrm{m}^{\prime}$.

\section{EXPERIMENT I}

In the present study, the difference between the perceived and physical distance of the two ends $(j$ and $\mathrm{k})$ of a vertically extended object was varied by modifications of the size (perspective) cue of distance. The stimulus object was tapered to produce the impression that the small end was more distant than the large end. In Experiment I, this perspective cue was either in the direction of or opposite to the physical slant (a physical tilt in depth), depending upon whether the smaller end of the object was the higher or lower end in the visual field. In Experiment II, since the object was physically always in a frontoparallel plane, the use of a tapered object always resulted in a perceived slant in depth that was different from the physical slant.

\section{Method}

Observers. The Os were undergraduate students ( 42 men and 30 women) with an average age of 19 years. All had a visual acuity of at least $20 / 20$ in both eyes, as measured on the near and far positions of a Keystone orthoscope, and a stereoacuity of at least $30 \mathrm{sec}$ of arc. Fourteen Os wore glasses and 15 wore contact lenses.

Apparatus. The situations in which only a tapered stimulus was presented and was viewed with a moving head are called the experimental situations. The observation position for the experimental situations was located in a totally dark observation booth and consisted of a head- and chinrest mounted on rollers, so that $O$ could move his head in the head-and chinrest repetitively right and left through a distance of $13.1 \mathrm{~cm}$. The observation in the experimental situations was always monocular, with the left eye of $O$ covered with an eye patch. The stimulus object corresponding to $\mathrm{jk}$ of Fig. 2 was a uniformly tapered electroluminescent surface, $80 \mathrm{~cm}$ long and white in color, with 
Table 1

Verbal Reports from Experiment I of Distance, $D_{v}$ (Converted to Centimeters), Obtained from the Calibration and Experimental Situations, and the Results from Transforming the Second by the First Into Perceived Distances, $D^{\prime}$

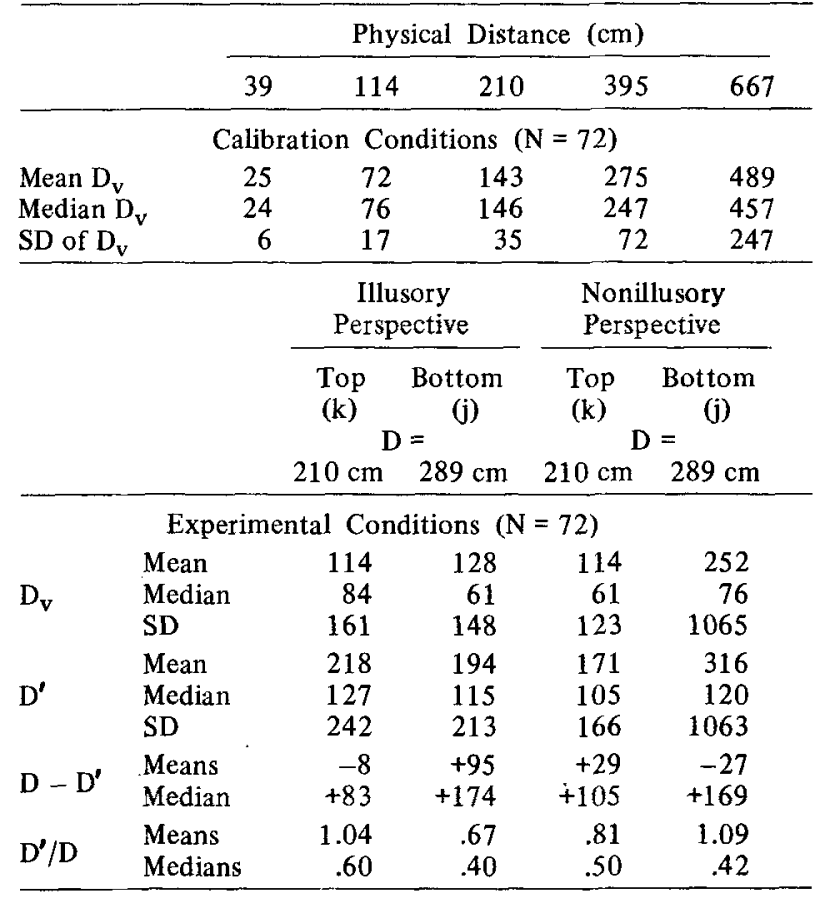

the large and small ends 2.5 and $.4 \mathrm{~cm}$ wide, respectively. This was physically slanted in depth $80 \mathrm{deg}$ from $O$ 's frontoparallel plane, with the upper (top) end always $210 \mathrm{~cm}$ and the lower (bottom) end always $289 \mathrm{~cm}$ from O's eye (as measured from the midpoint of the lateral head motion). The middle of the tapered object was at the height of O's eyes. This stimulus had a brightness of $.4 \mathrm{fL}$ and was the only object visible in an otherwise totally dark surround. The smaller end was the upper end in one condition and the lower end in another. When the smaller end was the upper end, the perspective cue was such as to make the upper end appear more distinct than the lower end. Since this perceived slant in depth was opposite to the physical slant in depth, this condition is called the "illusory perspective." When the upper end was the larger end, the direction of slant in depth from the perspective cue was in agreement with the direction of the physical slant. This condition is called the "nonillusory perspective." The stimulus was visible to $O$ only when the $E$ raised a shutter located near the observation position. Communication between $\mathrm{O}$ and $\mathrm{E}$ was by means of a microphone and headphones. To pace O's head movements in the experimental conditions, a click from an electronic metronome was presented to $O$ every $1.4 \mathrm{sec}$ through the headphones. Between presentations (trials), white noise was presented to mask any sound involved in changing the stimulus conditions.

The situation in which many cues of distance were available in order to obtain calibration equations for transforming verbal reports of distance to perceived distance is called the calibration situation. The observation position and alley for the calibration situation, like that for the experimental situations, contained a head- and chinrest, but for the calibration, the headrest was always immobile. Extending from the observation position in the calibration situation was an alley with a floor $(99.5 \mathrm{~cm}$ wide and $747 \mathrm{~cm}$ long) located $32 \mathrm{~cm}$ below the level of $O$ 's eyes and covered with white cloth. The walls of the alley were formed by black curtains. White numbered squares $(10 \mathrm{~cm}$ on a side) were located at $39,114,210,395$, and $667 \mathrm{~cm}$ from $O$, with the squares placed alternately to the right and left of the midline of the floor. The alley was illuminated by a row of overhead lights, and was always observed binocularly.

Procedure. The experimental situations were always presented before presenting the calibration situation. Before presenting any experimental situation, the tasks of $O$ were explained and $O$ was given practice in using the head-movement apparatus. A series of head movements was always started from the extreme right or left position. $O$ was instructed to move the head- and chinrest by moving his head so that the headrest contacted a stop at either end of the movement simultaneously with the occurrence of a click. O was informed that the stimulus might or might not appear to be slanted in depth, that it might or might not appear to move with head motion, and that his reports were to indicate appearances regardless of any notions he might have about the physical motions or physical distances of the stimulus.

For each stimulus presentation, whether illusory or nonillusory, $O$ completed three tasks in the following order. (a) With head stationary, $O$ reported verbally in feet or inches, or in some combination of both, the perceived distance of one of the two ends of the stimulus as identified by $E$. (b) With the metronome sounding, $O$ moved his head in time with the clicks, keeping his gaze fixed on the end of the stimulus whose apparent distance he just reported. Following four head movements, the shutter was closed, and $\mathrm{O}$ reported verbally whether the end that he had been fixating appeared to remain stationary or to move right and left as he moved his head. (c) If the end was reported to move, $O$ was asked whether the direction of the apparent motion was the same as, or opposite to, the direction of the head movements, and to indicate in feet or inches, or ir. some combination of both, the magnitude of this apparent motion.

These tasks were then repeated for the other (remaining) end of the stimulus. All Os completed the tasks involving both the illusory and nonillusory perspective. The order in which the illusory and nonillusory perspectives were presented and the order in which reports were obtained for the two ends of the stimulus were counterbalanced between Os.

In the calibration situation, $O$ verbally reported in feet or inches, or in some combination of both, the apparent distance, from his eyes, of each of the numbered squares on the alley floor. All five squares were presented simultaneously along the alley floor, with each $\mathrm{O}$ reporting the perceived distances of the different squares in a different random order.

\section{Results}

Perceived Distance. The verbal reports of distance $\left(D_{v}\right)$ obtained from the calibration situation are shown in the upper portion of Table 1 . Under the assumption that physical distances (D) in the calibration field were correctly perceived, the relationship between $D_{v}$ and perceived distance $\left(D^{\prime}\right)$ in the experimental conditions was determined for each $O$ by computing the linear line of best fit for the power function relating $D_{v}$ and $D$ obtained in the calibration situation. From the average coefficient and exponent from these individual power functions, the power function relating $D_{v}$ and $D$ was $D_{v}=.6 D^{1.04}$. The individual calibration equations were used to transform the verbal reports of distance obtained from each $\mathrm{O}$ in the experimental conditions to perceived distances. The means, medians, and standard deviations of the perceived distances of the two ends of the stimulus object obtained in this manner and D - $D^{\prime}$ and $\mathrm{D}^{\prime} / \mathrm{D}$ calculated from these means and medians also are shown in Table 1. Since the distributions often were skewed, medians, rather than means are considered to best represent the data. 
From the median D - D' results of Table 1, with both the illusory and nonillusory perspective, both ends of the object tended to be perceived as less distant from $O$ than the physical distances to the ends. It follows from Eq. 2 that perceived common motion should have been present with both the illusory and nonillusory perspectives and that this perceived common motion should have occurred in the same direction as the head motion. From the median $D^{\prime} / D$ results of Table 1 , $D_{k}^{\prime} / D_{k}>D_{j}^{\prime} / D_{j}$ for both the illusory and nonillusory perspective orientations. It follows from Eq. 3, that for both of these perspective conditions, $m_{j}^{\prime}$ should have been greater than $m_{k}^{\prime}$. In other words, from the $D^{\prime}$ data, according to Eq. 2, perceived common motion should have occurred with this perceived common motion indicated by + values of both $m_{j}^{\prime}$ and $m_{k}^{\prime}$, and, according to Eq. 3, perceived relative motion should have occurred with this perceived relative motion indicated by larger values of $m_{j}^{\prime}$ than of $m_{k}^{\prime}$. Also, since the difference between $D_{k}^{\prime} / D_{k}$ and $D_{j}^{\prime} / D_{j}$ usually was greater for the illusory than for the nonillusory perspective (see Table 1), it follows from Eq. 3 that the magnitude of the apparent relative motion should have been greater with the illusory as compared with the nonillusory perspective.

Perceived Motion. The distributions of reports of perceived motion are skewed, and appreciable numbers of zero reports (no perceived motion) are present in the distributions. Thus, neither the mean nor the medians adequately represent the central tendencies of these distributions. It was decided, therefore, to summarize the perceived motion data in the manner shown in Table 2 . The left column of Table 2 designates intervals of magnitude for classifying the reports. The remaining columns of Table 2 indicate the number of reports which fall within these intervals for each of the four distributions (illusory, top; illusory, bottom; nonillusory, top; and nonillusory, bottom). The frequency of,$- ; 0$, and + reports are summarized at the bottom of Table 2 for each of the four distributions. It will be noted that for both the illusory and nonillusory perspective conditions, $m_{k}^{\prime}$ was very often negative and $\mathrm{m}_{\mathrm{j}}$ was most often positive. Thus, consistent with the predicted difference between $m_{j}^{\prime}$ and $m_{k}^{\prime}$ from Eq. 3 and the $D^{\prime} / D$ data of Table 1 , it was found that usually $m_{j}^{\prime}-m_{k}^{\prime}>0$. A more direct test of the application of Eq. 3 to the obtained data is to compare the sign of the right side of Eq. 3 determined from the $D^{\prime} / D$ data with the sign of the left side of Eq. 3 determined by the $\mathrm{m}^{\prime}$ data. In agreement with Eq. 3, it was found that the sign on the two sides of this equation was the same for 62 out of 72 Os with the illusory perspective and for 50 out of $72 \mathrm{Os}$ for the nonillusory perspective. Also, consistent with the $D^{\prime} / D$ results, the magnitude of $m_{j}^{\prime}-m_{k}^{\prime}$ was significantly larger (at the .01 level) using the Wilcoxon test $(Z=2.31)$ for the illusory as compared with the nonillusory perspective condition. It can be concluded that perceived relative motion as defined by Eq. 3
Table 2

Frequency of Reports from Experiment I of Perceived Motion Converted to Centimeters

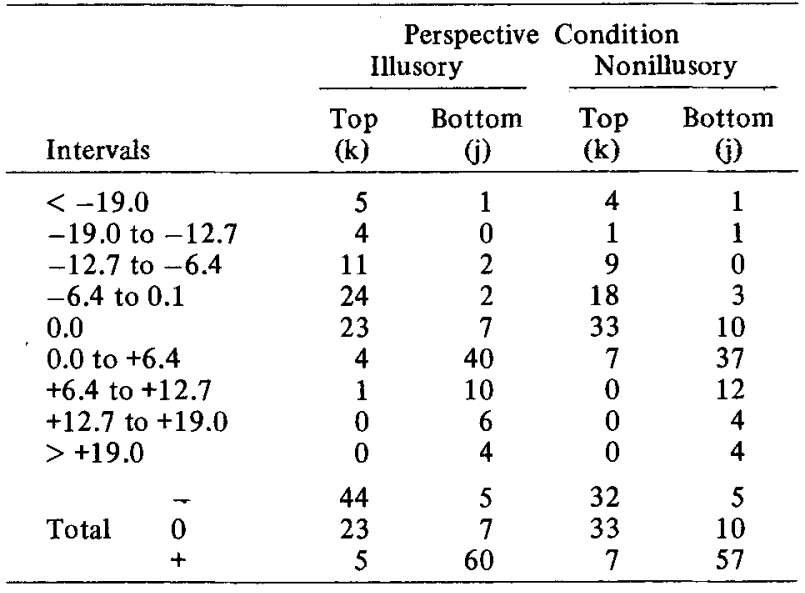

occurred in the direction expected from the $D^{\prime} / D$ data, and also as expected from the $\mathrm{D}^{\prime} / \mathrm{D}$ data, the amount of perceived relative motion was greater with the illusory than with the nonillusory perspective.

Although the $D^{\prime} / D$ results are consistent with the perceived relative motion expected from Eq. 3, the perception of common motion expected from the D - D' data using Eq. 2 did not occur. In order for the data of Table 2 to reflect perceived common motion consistent with the data of Table 1 , the perceived motion of both the top and bottom of the stimulus should have been positive. Also, considering the individual data from both the illusory and nonillusory perspective conditions, it was found that only four Os reported that the perceived motion of the top and bottom were both positive or both negative when the $D-D^{\prime}$ of the top and bottom were both positive or both negative. The perception of common motion expected from Eq. 2 did not occur.

\section{Discussion}

The success of Eq. 3 but not of Eq. 2 in predicting the perceived motion data from the perceived distance data possibly can be understood in terms of the inability of $O$, despite the instructions, to consider the apparent motion of one end independently of the apparent motion of the other end. It is suggested that the perception that one end was moving differently than the other end may have dominated O's response to such an extent that he tended to neglect the apparent common motion when giving his report of apparent motion. In agreement with this possibility, Wallach (1959) has noted that in a situation in which both perceived relative and perceived common motion occur, $\mathbf{O}$ must often be prompted before he will report the common motion. It is as if the perception of relative motion is so impressive that the common perceived motion is often neglected. To the Es, both the relative and common components of perceived motion were observable. It is suggested that 
the occurrence of apparent common motion probably should be reexamined, using Os more sophisticated than those of the present study.

\section{EXPERIMENT II}

Experiment II was designed to test further the application of Eq. 3 to the perception of the motion of a stationary object resulting from head motion. This seemed desirable for two reasons. First, in Experiment I, reports of perceived motion were obtained for $\mathrm{j}$ and $\mathrm{k}$ separately. The occurrence of perceived relative motion rather than being directly reported was inferred from the reports by the application of Eq. 3 to the $\mathrm{m}^{\prime}$ and $\mathrm{D}^{\prime} / \mathrm{D}$ data. A more direct test of Eq. 3 would involve applying this equation to a situation in which $\mathrm{O}$ directly indicated the apparent relative motion of the parts of the stimulus. Second, the perception of relative motion in Experiment I inferred from Eq. 3 is consistent with the differential motion on the eye of the lower end (j) of the stimulus relative to the upper end $(k)$ as the head was moved laterally. But Eq. 3 is not limited to situations in which relative retinal motion is present when the head is moved. In Experiment II, since the vertically extended object was always presented physically in a frontoparallel plane, lateral head motion did not result in relative motion on the eye. Nevertheless, according to Eq. 3, the use of an illusory perspective should result in apparent relative motion with head motion under these conditions also.

The direct measure of apparent relative motion in Experiment II consisted of $\mathrm{O}$ reporting the apparent magnitude and direction of the rotation ( $\alpha^{\prime}$ in Fig. 2) of the physically frontoparallel, but apparently slanted, object. Two illusory perspectives were used. In one of these, the top end was at a greater perspective distance than the bottom end, and in the other, the direction of the perspective was reversed. Under these conditions, if the perspective cue results in the top end of the object appearing more distant than the bottom end, from Eq. 3, the object should appear to rotate clockwise as the head is moved from right to left (see Fig. 2). On the other hand, whenever the perspective results in the top end of the object appearing to be less distant than the bottom end, the direction of apparent rotation relative to the direction of head motion should be reversed.

Since, in Experiment II, the object, jk, was always physically in a frontoparallel plane, Eq. 3 reduces to

$$
m_{j}^{\prime}-m_{k}^{\prime}=A\left(D_{k}^{\prime}-D_{j}^{\prime}\right) / D
$$

or

$$
\mathrm{m}_{\mathbf{j}}^{\prime}-\mathrm{m}_{\mathbf{k}}^{\prime}=\operatorname{Ad}_{\mathbf{j k}}^{\prime} / \mathrm{D},
$$

where $d_{j k}^{\prime}$ is the difference in the perceived distance between the top and bottom and $D$ is the physical distance of both $\mathrm{j}$ and $\mathrm{k}$ from $\mathrm{O}$. In order to relate the perception of relative motion indicated by $\alpha^{\prime}$ to $m_{j}^{\prime}-m_{k}^{\prime}$ in Eq. 5, it is necessary to determine the perceived length, $S^{\prime}$ of the object, jk, in the plane of apparent orientation, $\mathrm{j}_{1}^{\prime} \mathrm{j}_{2}^{\prime} \mathrm{k}_{2}^{\prime} \mathrm{k}_{1}^{\prime}$ (see Fig. 2). A reasonable approximation of the relation between $\mathrm{m}_{\mathrm{j}}^{\prime}-\mathrm{m}_{\mathrm{k}}^{\prime}$ and $\alpha^{\prime}$ is that

$$
\alpha^{\prime}=\left(m_{j}^{\prime}-m_{k}^{\prime}\right) / S^{\prime},
$$

where $\alpha^{\prime}$ is expressed in radians. Combining Eqs. 5 and 6 yields

$$
\alpha^{\prime}=\operatorname{Ad}^{\prime} / \mathrm{DS}^{\prime}
$$

Since A, D, and $\mathrm{S}^{\prime}$ are always positive, the sign of $\alpha^{\prime}$ is determined by the sign of $d^{\prime}$. If the top of the object is at a greater perceived distance than the bottom ( $\mathrm{d}^{\prime}$ positive), the apparent rotation $\alpha^{\prime}$ should be in a clockwise (positive) direction when the head is moved from Position 1 to Position 2. If the top of the object is at a smaller perceived distance than the bottom ( $\mathrm{d}^{\text {' }}$ negative), $\alpha^{\prime}$ should be counterclockwise (negative) as the head is moved from Position 1 to Position 2. To test the validity of Eq. 3 by means of Eq. 7, reports of $S^{\prime}$ and $d^{\prime}$ (with $d^{\prime}$ obtained from the difference in $D^{\prime}$ to the ends of the stimulus) were compared with the obtained values of $\alpha^{\prime}$ using Eq. 7 .

\section{Method}

Observers. The Os were 60 undergraduate students ( 25 men and 35 women) with an average age of 18 years, none of whom had participated in Experiment I. All Os had a visual acuity of at least $20 / 20$ in the right eye, as measured at the near position on a Keystone orthoscope. Twenty-two Os wore glasses and 8 wore contact lenses.

Apparatus. The observation booth and head- and chinrest was the same as in Experiment I, except that in Experiment II the lateral movement of the headrest assembly was $20 \mathrm{~cm}$. The metronome rate was one click every $0.7 \mathrm{sec}$ in Experiment II.

A rear-projection screen, oriented in $O$ 's frontoparallel plane throughout the experiment, was located $70 \mathrm{~cm}$ from $O$ 's eyes. The stimulus object projected on this screen contained an adjustable perspective cue. Except for the stimulus object, the screen and the remainder of the visual field were totally dark. The stimulus on the screen was produced by placing an appropriately masked transparency $45 \mathrm{~cm}$ behind the screen and $14 \mathrm{~cm}$ in front of a point source of light. The point source, the center of the transparency, and the center of the projected image of the transparency on the screen were at the level of O's eyes. By means of a reversible motor operated by either $\mathrm{E}$ or $\mathrm{O}$, the transparency could be slanted in depth in either direction from the frontoparallel plane by rotating it around a horizontal axis through its midpoint. The depth slant of the transparency varied the distances of its top and bottom from the point source, thereby varying the gradient of relative physical sizes (the perspective cue) from the top to the bottom of the stimulus on the frontoparallel projection screen. The three conditions of this perspective cue are illustrated in Fig. 3. In A in Fig. 3, the transparency was vertically oriented and the stimulus on the screen had a perspective that would be expected to make all portions of the object appear frontoparallel. In $\mathrm{B}$ and $\mathrm{C}$ in Fig. 3, the transparency was slanted in depth so as to produce a perspective cue that would be expected to make the top end appear nearer or farther than the bottom end, respectively. It will be noted that the height of the image on the screen, as well 

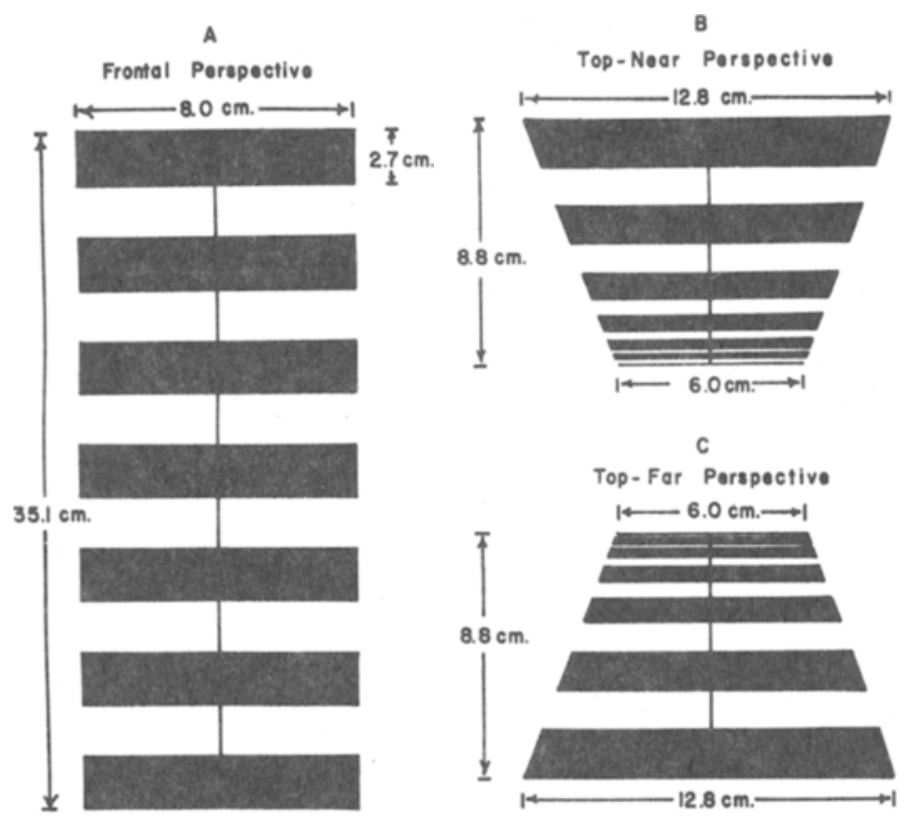

as the perspective cue, varied with the physical slant of the transparency. Three conditions of perspective cue were used in the experiment. Two of these were adjusted by $E$ to predetermined values so as to produce the two perspective conditions on the rear-projection screen illustrated by $B$ and $C$ in Fig. 3. The condition illustrated by $B$ in Fig. 3 will be called the top-near perspective, and the conditions illustrated by $\mathrm{C}$ in Fig. 3 will be called the top-far perspective. According to Eq. 7, $\alpha^{\prime}$ will be negative for the top-near perspective and positive for the top-far perspective. The third perspective condition was obtained by having $O$ adjust the physical slant of the transparency to the criterion that the object appeared to $O$ to be frontoparallel. On the average, it would be expected that this adjustment would result in the frontoparallel perspective shown by A in Fig. 3, and this condition will be called the frontal perspective. To avoid any scattered light, the stimulus on the screen was dim, with a luminance of $.0024 \mathrm{fL}$. No object or surface except the stimulus object was visible during the experiment. The $O$ was dark-adapted for $1 \mathrm{~min}$ before being presented with any of the perspective conditions, so that the object on the screen was clearly visible.

A rod, $21 \mathrm{~cm}$ long and located on O's right at waist level, was used by $\mathrm{O}$ to indicate the magnitude of the perceived rotation of the stimulus. The rod could be rotated about its midpoint and was mounted on a surface which $O$ could slant in depth to match the plane of apparent slant of the stimulus (the plane defined by $j_{1}^{\prime} j_{2}^{\prime \prime} k_{2}^{\prime} k_{1}^{\prime}$ in Fig. 2). A potentiometer fixed to the pivot axis of the rod was connected to a regulated voltage source and digital voltmeter. The voltmeter reading varied linearly with the angle of rotation of the rod, allowing $E$ to record O's rotational adjustments from a position outside the observation booth. A microphone and headset permitted $\mathrm{O}$ and $\mathrm{E}$ to communicate and also provided for presentation of the metronome and white noise, as in Experiment I.

Procedure. Before being presented with the stimulus, $O$ became familiar with the operation of the headrest assembly and was instructed as to his tasks using a photograph of the stimulus to illustrate the manner in which the vertical center line of the stimulus might appear to rotate as $O$ moved his head. As in Experiment I, Os were instructed to base their reports on appearances rather than what they thought the physical condition of the stimulus might be. The experiment consisted of three trials, each with the stimulus on the physically frontoparallel screen presented with a different perspective, i.e., the top-near, top-far, or frontal perspective, depending upon a predetermined order counterbalanced between Os. Each $O$ completed the tasks in the following order for each trial. (a) With the headrest stationary at the extreme right or left side of movement, $O$ reported verbally in feet or inches, or in some combination of both, the perceived distance to the top end and, following this, to the bottom end of the stimulus. (b) Again with the head stationary, $O$ reported in feet or inches, or in some combination of both, the apparent size $\left(S^{\prime}\right)$ of the stimulus, i.e., the apparent distance along the stimulus (from the top to the bottom) in the plane of the apparent slant of the stimulus. (c) Fixating on the center line of the stimulus, $O$ moved his head left and right in time with the metronome. Following four head movements, the, viewing aperture was closed and, with the booth lights turned on, $O$ oriented the rod to indicate the extreme amount that the vertical centerline of the object appeared to rotate in a clockwise, and following this in a counterclockwise, direction. Before making this response to perceived rotation, $\mathrm{O}$ adjusted the depth inclination of the rod and the surface on which the rod was mounted to be equal to the apparent slant in depth of the stimulus object. (d) If $O$ indicated that the object had appeared to rotate, the apparent direction of the rotation with respect to the direction of O's head motion was determined by presenting the stimulus again and having $O$ report the direction in which the stimulus appeared to rotate as he made one more head movement.

For all presentations, the stimulus was initially in a frontoparallel perspective. For the top-near and top-far perspective conditions, $O$ observed the stimulus while $E$ rotated the transparency to produce the required perspective condition on the frontoparallel screen. For the frontal perspective condition, $\mathrm{O}$ used a switch at the observation position to adjust the physical slant of the transparency until both ends of the stimulus appeared to be the same distance from his eyes. The frontal perspective constituted a control condition in which no perceived rotation was expected, since with this condition the apparent depth between the two ends of the stimuli was in agreement with the zero amount of physical depth.

An opaque eye patch was worn over the left eye of all Os throughout the experiment. Due to the length of the experiment, a distance calibration condition was not included. Distance calibration is less important here than in Experiment $I$ 
Table 3

Verbal Reports from Experiment II of the Perceived Distance to the Top End of the Stimulus ( $D_{k}^{\prime}$ ), the Perceived Distance to the Bottom End of the Stimulus $\left(D_{j}^{\prime}\right)$, and the Perceived Size of the Stimulus $\left(S_{j k}^{\prime}\right)$

\begin{tabular}{|c|c|c|c|c|c|c|c|c|c|}
\hline & \multicolumn{3}{|c|}{ Top Far Perspective } & \multicolumn{3}{|c|}{ Frontal Perspective } & \multicolumn{3}{|c|}{ Top Near Perspective } \\
\hline & Mean & Median & SD & Mean & Median & SD & Mean & Median & SD \\
\hline $\mathrm{D}_{\mathrm{k}}^{\prime}$ & 101 & 61 & 163 & 51 & 30 & 54 & 53 & 29 & 192 \\
\hline $\mathrm{D}_{\mathrm{j}}^{k_{k}}$ & 52 & 30 & 124 & 51 & 30 & 54 & 140 & 61 & 467 \\
\hline $\mathbf{S}_{\mathbf{j k}}^{\prime}$ & 71 & 30 & 139 & 37 & 30 & 26 & 105 & 30 & 391 \\
\hline $\mathrm{d}_{\mathrm{jk} \mathrm{k}}$. & +49 & +31 & & 0 & 0 & & -87 & -32 & \\
\hline
\end{tabular}

Note-All reports converted to centimeters.

since the sign of $d^{\prime}$, unlike the sign of $D-D^{\prime}$, could not be affected by a multiplicative calibration constant.

\section{Results}

Table 3 gives the verbal reports of perceived distance to the top $\left(D_{k}^{\prime}\right)$ and bottom $\left(D_{j}^{\prime}\right)$ end of the stimulus and the reported length $\left(S_{j k}^{\prime}\right)$ of the stimulus. As in Experiment $I$, medians rather than means are considered to best represent the data. The $d_{j k}^{\prime}$ values in the bottom row of Table 3 found by subtracting $D_{j}^{\prime}$ from $D_{k}^{\prime}$ indicate that, as was expected, the top end appeared to be the more distant end with the top-far perspective and to be the less distant end with the top-near perspective. It follows from Eq. 7 that as $D$ moved his head from right to left, the stimulus should have appeared to rotate clockwise with the top-far perspective, to rotate counterclockwise with the top-near perspective, and to remain stationary with the frontal perspective. The occurrence and directions of apparent rotation $\left(\alpha^{\prime}\right)$ are shown in Table 4 and are consistent with these expectations. The median $\alpha^{\prime}$ for Os who saw rotation in the predicted direction was $36 \mathrm{deg}$ clockwise for the top-far perspective, $27 \mathrm{deg}$ counterclockwise for the top-near perspective, and $0 \mathrm{deg}$ for the frontal perspective. The effect of perspective on perceived rotation was significant beyond the .01 level by a Friedman two-way analysis of variance $\left(\chi^{2}=55.8\right)$. A Pearson product-moment correlation coefficient was computed between the $\alpha^{\prime}$ and $\mathrm{Ad}^{\prime} / \mathrm{DS}^{\prime}$ with each of the three perspective conditions for each $O$. Correlations could not be computed for 10 Os because all values of $\alpha^{\prime}$ from these Os were zero. The mean, median, and SD of the distribution of correlations for the remaining $50 \mathrm{Os}$ was $.78, .87$, and .34 , respectively. Forty-four of the correlations were greater than .75 . Thus, there was a strong tendency for $\alpha^{\prime}$ to vary with $\mathrm{Ad}^{\prime} / \mathrm{DS}^{\prime}$ in the direction predicted from Eq. 7.

The $\alpha^{\prime}$ and $\mathrm{Ad}^{\prime} / \mathrm{DS}^{\prime}$ data can be used to determine rank order data to further test Eq. 7. For this purpose, the magnitudes of $\alpha^{\prime}$ and $\mathrm{Ad}^{\prime} / \mathrm{DS}^{\prime}$ were ranked across the three perspective orientations for each $O$. The average rank order data is plotted in Fig. 4. From Fig. 4, the tendency for $\alpha^{\prime}$ to be an increasing function of $\mathrm{Ad}^{\prime} / \mathrm{DS}^{\prime}$ (as would be expected from Eq. 7) is clear. The results from Experiment II indicate that a discrepancy between the physical and perceived distances of physically stationary points or parts of objects will result in perceived relative motion even though a differential displacement of the points or parts on the eye is not vresent during head motion.

\section{GENERAL DISCUSSION}

From the present study, the $D^{\prime} / D$ differences between physically stationary points of stimulation is an important factor in determining the relative apparent motion (or lack of relative apparent motion) resulting from head motion. This factor is of consequence even in a situation in which the head motion does not result in a differential motion of the stimuli on the eye. It seems that any explanation of this relative apparent motion (or of the constancy of relative visual direction despite head motion) solely in terms of the relation between efference and reafference information is inadequate. In order to explain the present results in terms of the reafference theory, it seems necessary to postulate that the relation between efference and reafference can be modified by the perceived distances of the stimuli.

It is suggested that the lack of common motion in the analysis of Experiment I using Eq. 2 was due to the tendency for common motion to be unnoticed with naive Os in the presence of apparent relative motion. Consistent with this interpretation, it has been found that Eq. 2 can predict the direction of motion resulting from head motion when only one point of light is present, i.e., in the absence of apparent relative motion (Gogel \& Tietz, 1972). Considering both of these studies together, it seems that Eqs. 1 through 7 are useful in predicting the occurrence and direction of the apparent motion resulting from head motion. It should be noted, however, that the derivation of Eqs. 2 through 7 requires the assumption that $\mathrm{A}^{\prime}=\mathrm{A}$ and $\phi_{\mathrm{T}}^{\prime}=\phi_{\mathrm{T}}$ for each of the

Table 4

Number of Os Who Reported Clockwise Rotation, No Rotation, or Counterclockwise Rotation in Experiment II Under Each of the Perspective Conditions

\begin{tabular}{lccc}
\hline $\begin{array}{c}\text { Direction of } \alpha^{\prime} \text { With } \\
\text { Right to Left } \\
\text { Head Motion }\end{array}$ & \multicolumn{3}{c}{ Perspective Condition } \\
\cline { 2 - 4 } & Top Far & Frontal & Top Near \\
\hline Clockwise & 42 & 4 & 2 \\
Stationary & 16 & 53 & 32 \\
Counterclockwise & 2 & 3 & 26 \\
\hline
\end{tabular}




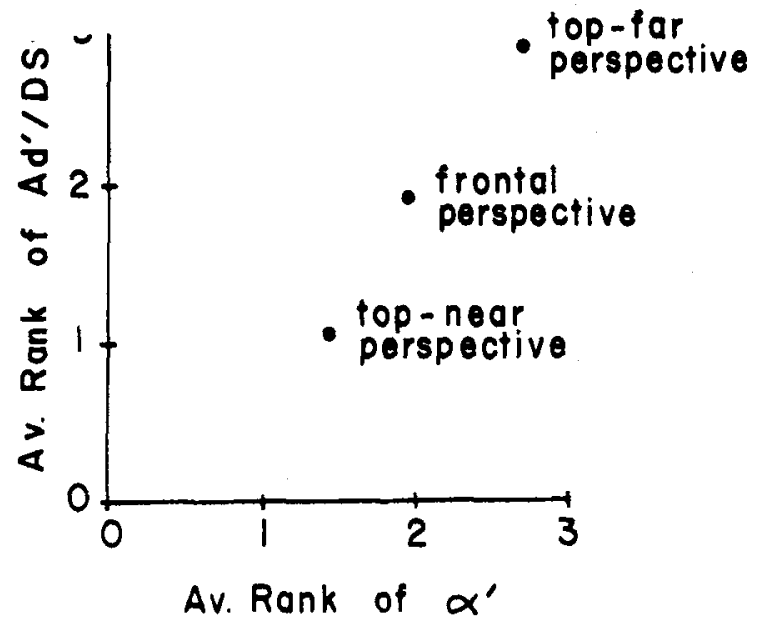

Fig. 4. The relation between the perceived rotation, obtained directly by the adjustment method and computed from perceived depth and perceived size using Eq. 7 .

points or object parts being considered. The general point of view of these studies, however, is expressed by Eq. 1 and therefore does not depend upon these simplifying assumptions. For cases in which these assumptions are not justified, the more complete description of the factors determining perceived motion, as expressed by Eq. 1, must be applied.

The perception of relative motion between parts of a physically stationary scene by a moving $O$ has been reported in a number of circumstances (Ittelson, 1960; Miller, 1962; Jerrison, 1967; Gregory, 1970). For example, Gregory (1970, pp. 126-131) describes a case in which a concave mask of a human face will appear to turn to follow a moving $O$. A necessary condition for this phenomenon is that the face appear normal (protruding rather than concave). This is an instance in which the parts of the object (mask) differ in their physical and perceived distances with the apparent rotation of the face consistent with the results of the present study. On the other hand, it would be predicted that if a convex (protruding) object appeared concave, the object would appear to turn in the direction opposite to the direction of motion of the 0 . The same reason (differences between $D^{\prime} / D$ of the various parts of the scene) can be used to explain the apparent motion that occurs with head motion when viewing a stereogram in which different parts of the scene appear at different distances although these parts are physically equidistant. It also follows that whenever motion of the head produces a perceived relative motion of the parts of a scene, these parts are not perceived to be at their physical distances. It is often found, for example, that if two objects at different physical distances are viewed while moving the head, the nearer object will appear to move relative to the farther object in a direction opposite to the direction of the head motion. From the present study, this perceived motion, rather than being caused by the relative displacement of the two objects on the retina, very likely is the result of perceiving the separation of the objects in depth as less than their physical depth. ${ }^{1}$ Indeed, it is expected from the present study that if the perceived depth between two objects were greater than the physical depth, the relative direction of the apparent motion of the two stationary objects with head motion would be reversed.

\section{REFERENCES}

Gogel, w. C. The sensing of retinal size. Vision Research, 1969, 9, 3-24.

Gogel, w. C. Equidistance tendency and its consequences. Psychological Bulletin, 1965, 64, 153-163.

Gogel, W. C., \& Tietz, J. D. Absolute motion parallax and the specific distance tendency. Perception \& Psychophysics, 1973, 13, 284-292.

Gregory, R. L. The intelligent eye. New York: McGraw-Hill, 1970 .

Hay, J. C., \& Sawyer, S. Position constancy and binocular convergence. Perception \& Psychophy sics, 1969, 5, 310-312. Ittelson, W. H. Visual space perception. New York: Singer, 1960.

Jerrison, $H$. J. Apparent motion of a vista: An illusion of perspective. American Journal of Psychology, 1967, 80, 448-453.

Miller, G. Space. In Psychology: The science of mental life. New York: Harper \& Row, 1962, Pp. 114-117.

Rock. I. The nature of perceptual adaptation. New York: Basic Books, 1966.

Wallach, H. The perception of motion. Scientific American, July $1959,2-6$.

Wallach, H., \& Kravitz, J. H. The measurement of the constancy of visual direction and of its adaptation. Psychonomic Science, 1965, 2, 217-218.

Wallach, H., Yablick, G. S., \& Smith, A. Target distance and adaptation in distance perception in the constancy of visual direction. Perception \& Psychophysics, 1972, 11, 3-34.

von Holst, $E$. Relations between the central nervous system and the peripheral organs. British Journal of Animal Behavior, $1954,2,89-94$.

\section{NOTE}

1. This underestimation of depth would be expected from research which indicates a prevailing tendency to see objects as equidistant with the reduetion in the perceived depth between objects from this tendency increasing as cues to distance are increasingly reduced (Gogel, 1965).

(Received for publication August 23, 1973; revision received February $27,1974$. ) 